\section{COXEM CO., LTD}

COXEM is a market-leading manufacturer of Scanning Electron Microscopes. We offer both bench-top SEM and console or full-size SEM with various optional features such as STEM and Cold Stage. COXEM is dedicated to serving all customers in a wide range of scientific fields with its high-quality products and optimized solutions.

\section{- EM-30N: Microscopy to the Power of "N" - Tabletop SEM}

The EM- $30^{\mathrm{N}}$ is the latest result of COXEM's steady investment in technology and development with a view to the era of nano-mechatronics. Producing clear, low noise images at high magnification, the EM- $30^{\mathrm{N}}$ also features our new Panorama mode, providing simple imaging of large areas at higher magnification. Full compatibility with market leading EDS suppliers delivers optimized performance.

Offering exceptional performance and outstanding value, the $\mathrm{EM}-30^{\mathrm{N}}$ will excel in all research areas and deliver superb results for the development and utilization of advanced technology.

High Resolution: the EM-30 ${ }^{\mathrm{N}}$ features completely re-designed imaging electronics for improved resolution with less noise at high magnification. The EM- $30^{\mathrm{N}}$ provides additional flexibility by allowing the operator to adjust accelerating voltage $(1-30 \mathrm{kV})$, working distance, and electron beam size.

Dual Display Mode: Delivered with both SE and BSE detectors, the EM-30N allows the operator to view either detector individually, side-by-side, or as a composite image for a better understanding of microstructure and chemistry.

Duplex Navigation : The NanoStation interface provides multiple means of navigation simply by clicking within any of the 3 different magnified views:

- A macro view to move from sample to sample or across areas of a large sample using the CCD Navi Cam (optical image) or Sample Holder map.

- A micro view for moving within the sample using the low magnification MiniMap SEM image with a Field of View (FOV) indicator.

- A nano view at the desired FOV allows fine movement by clicking in the image or using the Image Shift controls to make nanometer movements for perfect centering and alignment of samples.

Particle Analysis: Particle analysis can be done with various methods - both by image analysis and using EDS. Accurate analysis is ensured even for multi-component samples.

- EM-30LE: A CeB6 (cerium hexaboride) electron source version of the EM-30 $0^{\mathrm{N}}$ Table top SEM for increased resolution especially at the lower accelerating voltages typically used with biological and low atomic weight samples.

- CX-200Plus: NEW compact size floor model SEM that includes both SE and BSE detectors and a fully motorized 5-axis XYZRT stage as standard. A standard internal chamber view camera permits easy stage tilt and height adjustment for new users while the chamber provides numerous spare ports for future addition of EDS, EBSD, STEM, Cold Stage and Sample Transfer system (load/lock). A wide variety of EDS detector options are available including automated feature and particle analysis.

- CP-8000 (Ion Mill Cross Section Polisher): A compact economically priced sample preparation device to prepare cross sections of various materials using a gentle Ion Milling process.

- SPT-20: Our very popular and easy to use Ion Sputter Coater with a variety of targets such as $\mathrm{Au}, \mathrm{Pt}, \mathrm{Pd}$ and other metals.

As one of the fastest growing electron microscope brands, COXEM microscopes can be found all over the world in hundreds of global universities, labs, government research centers and industrial companies.
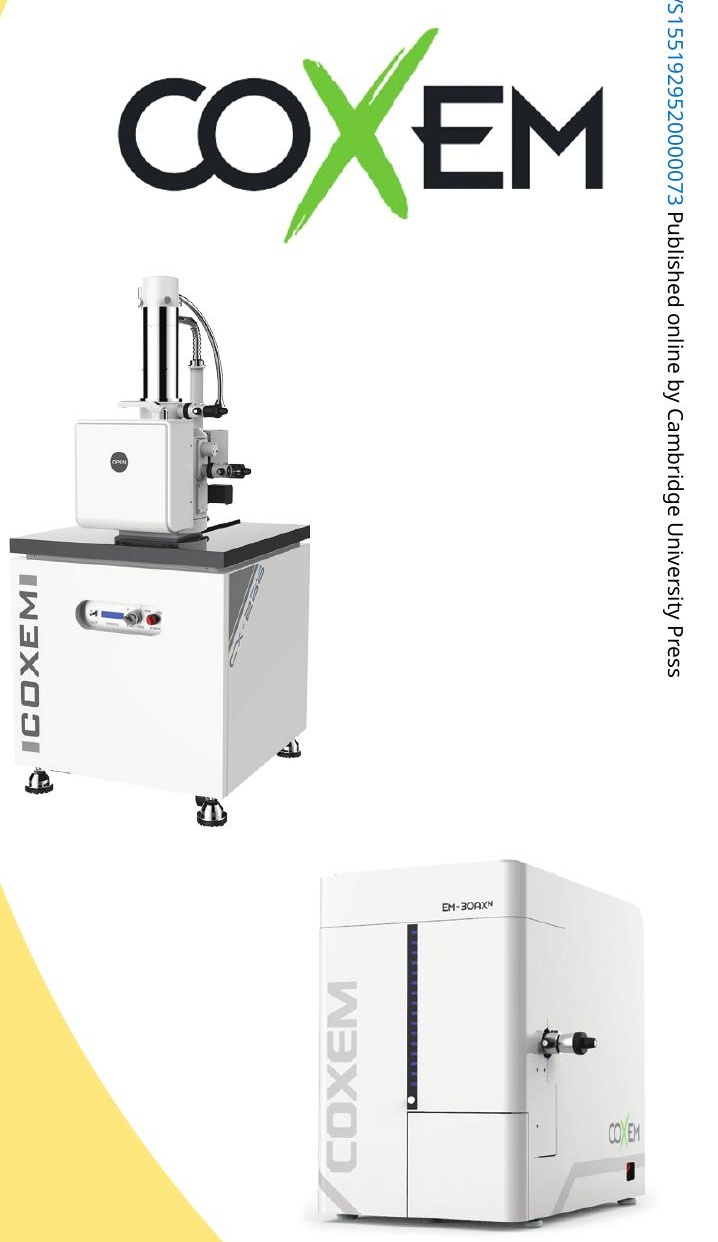

\section{How to find us}

\#201, Migun Techno World 1-cha, 199, Techno 2-ro, Yuseong-gu, Daejeon, Korea, 34025

Tel: +82.42 .861 .1686$

Fax: +82.42 .861 .1689$

Email: overseas@coxem.com

www.coxem.com

Worldwide locations.

To find your local distributor, please visit our website or email us. In North America, please call element Pi at 833-314-1593 or email Info@elementpi.com 